04

\title{
Отлипание электронов от молекул кислорода воздуха в интенсивном электрическом поле
}

\author{
(ㄱ Н.И. Буслеев, В.Л. Бычков, Л.П. Грачев, И.И. Есаков, А.А. Раваев \\ Московский радиотехнический институт РАН, \\ 117519 Москва, Россия \\ Te-mail: grachev@mrtiran.ru
}

(Поступило в Редакцию 25 октября 2016 г.)

Описываются результаты экспериментов по электрическому пробою воздуха в квазиоптическом СВЧ пучке с глубоко подкритическим уровнем исходного поля. Пробой инициировался цилиндрическим вибратором со сферически закругленными концами и вибратором с конически заостренным концом. Анализ опытных данных дает основание предположить, что необходимое наличие начальных электронов в пробойной области может обеспечиваться процессом их отлипания от молекул кислорода воздуха, который зависит от величины поля.

DOI: 10.21883/JTF.2017.09.44905.2079

\section{Введение}

В настоящее время рассматриваются различные варианты практического применения инициированных электромагнитным (ЭМ) вибратором газовых электрических разрядов в квазиоптическом СВЧ-пучке с глубоко подкритическим уровнем поля [1-3]. Здесь словосочетание „критический уровень поля“ $E_{\text {cr }}$ подразумевает его минимальный пробойный уровень в пространственнооднородной и непрерывной ЭМ волне [4]. Для зажигания разряда в подкритическом поле в СВЧ пучок параллельно вектору электрической составляющей исходной ЭМ волны $\mathbf{E}_{0}$ может быть помещен линейный цилиндрический ЭМ вибратор [5]. При этом амплитуда индуцированного поля на полюсах вибратора $E_{\text {pol может }}$ на несколько порядков превышать амплитуду исходного поля $E_{0}$. Это превышение реализуется в малой области, прилегающей к полюсным поверхностям вибратора. Для начала развития процесса электрического разряда в этой области поле $E_{\text {pol }}$ должно превышать величину критического поля пробоя $E_{\mathrm{cr}}$, и в ней должно быть некоторое число начальных свободных электронов $N_{e 0}$.

На практике при использовании для зажигания разряда СВЧ импульсов с длительностью $\tau_{\text {pul }}$ в десятые доли секунды требование наличия $N_{e 0}$ в пробойной области, как правило, не соблюдается. В этом случае в лабораторных исследованиях одну из полюсных поверхностей инициирующего разряд вибратора можно облучить синхронизированным с передним фронтом СВЧ импульса короткоимпульсным ультрафиолетовым (УФ) излучением, например, от сравнительно удаленного вспомогательного разряда [6]. Это УФ излучение за счет фотоэмиссии электронов с полюсной поверхности вибратора и обеспечивает их начальное наличие в пробойной области. В практических устройствах такая возможность технически может быть неосуществима. В то же время текущий экспериментальный опыт показал, что может реализоваться и другой способ обеспечения наличия в пробойной области требуемого числа $N_{e 0}$ [7]. В этих опытах один из полюсов вибратора конусно заострялся, и в этом случае пробой реализовывался и без вспомогательного УФ излучения. Объяснением этого явления может явиться процесс отлипания электронов от молекул кислорода в прилегающей к конической поверхности вибратора области воздуха в электрическом поле большой амплитуды.

В настоящей работе описываются результаты экспериментов по электрическому пробою воздуха в импульсном глубоко подкритическом исходном СВЧ поле при $E_{0} \ll E_{\mathrm{cr}}$. Пробой иницируется цилиндрическим ЭМ вибратором или со сферически закругленными концами, или вибратором, один конец которого конически заострен. Эксперименты проводились как при облучении вибраторов УФ излучением, так и без него. Полученные результаты обсуждаются и дают основание предположить, что необходимые для начала процесса развития СВЧ разряда свободные электроны в пробойной области могут появиться в результате их отлипания от отрицательно заряженных молекул кислорода воздуха в интенсивном СВЧ поле.

\section{Условия экспериментов и их результаты}

Эксперименты выполнялись на описанной в работе [8] установке с импульсным СВЧ излучением, имеющим длину волны $\lambda=12.3 \mathrm{~cm}$ при длительности импульсов $\tau_{\mathrm{pul}}=0.4 \mathrm{~s}$. В них, как показано на рис. 1 , линейно поляризованная СВЧ волна излучается вертикально вниз в СВЧ безэховую герметичную рабочую камеру. Давление воздуха в ней $p_{c}$ можно устанавливать от атмосферного значения до 10 Torr и измерять с точностью \pm 1.5 Torr. В СВЧ волну перпендикулярно ее волновому вектору $\mathbf{k}$ помещается плоский металлический лист-экран. На нем на пенопластовой подставке на расстоянии $h=\lambda / 4=31 \mathrm{~mm}$ от поверхности экрана 


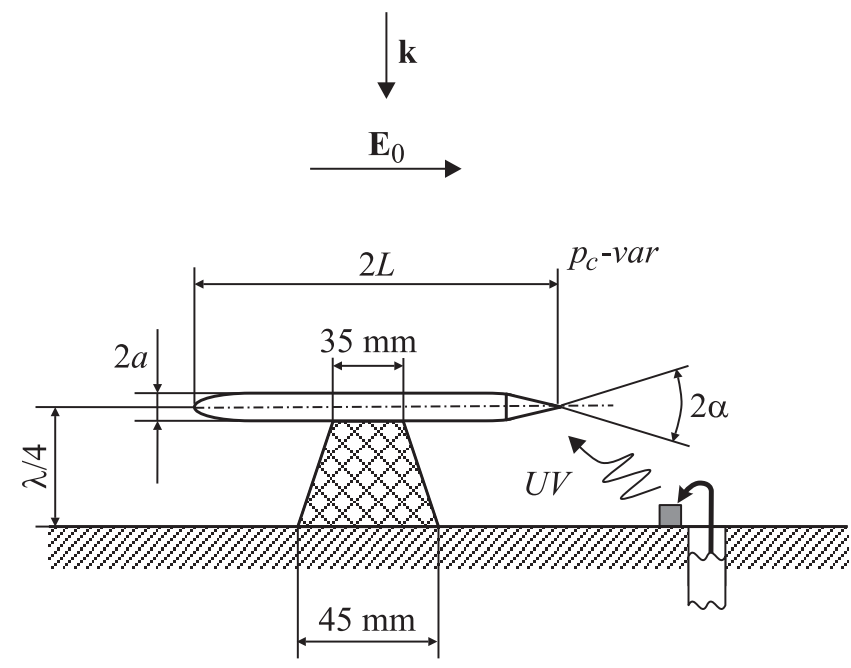

Рис. 1. Схема экспериментов.

в пучности падающей и отраженной ЭМ волн может помещаться цилиндрический алюминиевый вибратор, диаметром $2 a=5.6 \mathrm{~mm}$. В опытах использовались два варианта исполнения вибратора. В первом - оба конца вибратора сферически закруглялись, во втором - один его конец конически заострялся под углом $2 \alpha=20^{\circ}$. Длина вибраторов $2 L$ ступенчато уменьшалась от максимального значения $2 L \approx \lambda / 2$.

В опытах, как показано на рис. 1, мог использоваться вспомогательный разрядник - источник УФ излучения. Он обеспечивал зажигание искрового разряда длительностью в сотые доли секунды между внутренней жилой высоковольтного кабеля и поверхностью экрана. Включение этого разрядника синхронизировалось с передним фронтом СВЧ импульса. УФ излучение освещало поверхность одного из сферически закругленных концов вибратора или конически заостренный его конец. При этом для соблюдения неизменности геометрических факторов в пробойной области укорочение вибраторов производилось с противоположного конца.

В экспериментах предварительно была измерена амплитуда исходного поля $E_{0}$ при $h=\lambda / 4$. Для этого в предполагаемом месте расположения „рабочего“ ЭМ вибратора на экран помещалась пенопластовая прямоугольная стойка с размером поперечного сечения $20 \times 20 \mathrm{~mm}$. На ее верхнюю поверхность параллельно вектору $\mathbf{E}_{0}$ помещался короткий цилиндрический алюминиевый вибратор со сферически закругленными концами диаметром $2 a=5.6 \mathrm{~mm}$ и длиной $2 L=22.4 \mathrm{~mm}$, т.е. при отношении $2 L / 2 a=4$. У такого вибратора отношение $E_{\text {pol max }} / E_{0}=10$ [9]. В опыте определилось максимальное граничное давление воздуха в рабочей камере $p_{c}=p_{\text {br }}$, при котором при использовании вспомогательного разрядника этот вибратор инициировал СВЧ пробой. Измерения дали $p_{\mathrm{br}}=25$ Torr. Этому давлению в соответствии с работой [9] отвечает $E_{0}=180 \mathrm{~V} / \mathrm{cm}$.
В основной экспериментальной серии с двумя видами инициаторов также при каждом значении $2 L$ определялось граничное давление $p_{c}=p_{\mathrm{br}}$. При этом в опытах выяснилось, что в случае зажигания разряда поверхность вибратора существенно нагревается, что неконтролируемо влияет на величину $p_{\mathrm{br}}$. В связи с этим после фиксации горения разряда в конкретном СВЧ импульсе при необходимости следующее его включение осуществлялось лишь через временну́ю паузу, не меньшую минуты. В этом случае стабильность измеряемых $p_{\text {br }}$ выдерживалась.

Первоначально опыты выполнялись с вибратором со сферически закругленными концами. При этом выяснилось, что во всем диапазоне варьирования его длины $2 L$ без включения дополнительного разрядника он не инициировал СВЧ разряд. Инициация разряда осуществлялась только при облучении вибратора УФ излучением. Полученные экспериментально значения $p_{\mathrm{br}}$ при конкретных величинах $2 L$ в виде треугольников приведены на рис. 2 и соединены аппроксимирующей кривой. Она по характеру зависимости $p_{\mathrm{br}}(2 L)$ повторяет аналогичную экспериментальную зависимость, приведенную в работе [7]. Диапазон варьирования значений длины вибратора $\Delta 2 L$ в таком исполнении был определен по приведенным в этой работе результатам опытов с вибраторами с заостренным концом. Именно в этом диапазоне $\Delta 2 L$ длина последних была резонансной.

Результаты экспериментов с вибратором с заостренным концом также приведены на рис. 2. На нем значения $p_{\mathrm{br}}$, полученные с УФ излучением, показаны точками и соединены аппроксимирующей линией. Результаты же, полученные без УФ излучения, обозначены крестиками.

Эти эксперименты выполнялись в следующей последовательности.

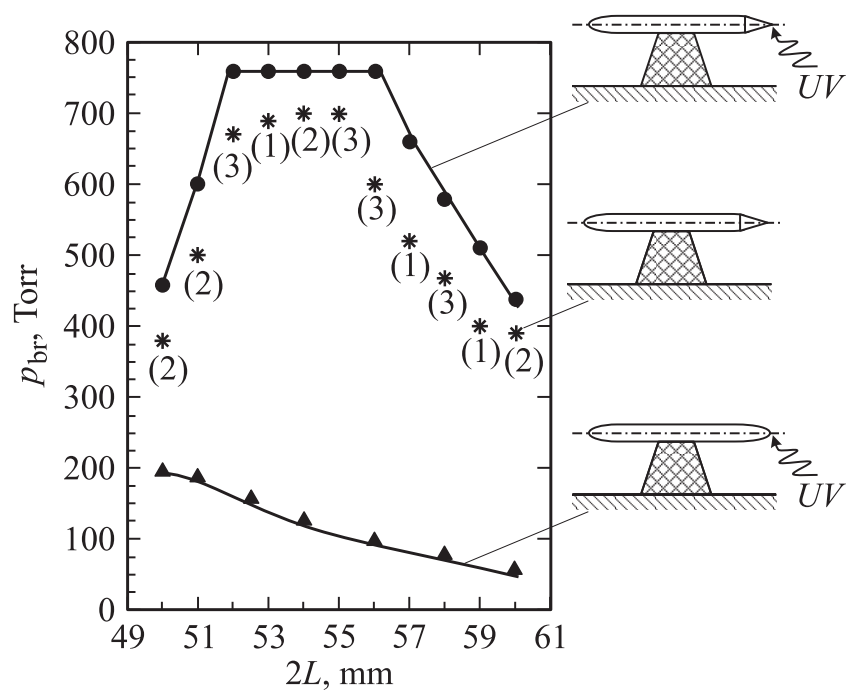

Рис. 2. Экспериментальные данные максимального пробойного давления воздуха при фиксированном уровне СВЧ поля в зависимости от длины вибратора. 
Первоначально в ЭМ пучок был помещен вибратор максимальной длины $2 L=60 \mathrm{~mm}$. Рабочая камера начинала плавно откачиваться с одновременным включением с частотой $1 \mathrm{~Hz}$ СВЧ импульсов и вспомогательного разрядника - источника УФ излучения. В опыте при $p_{c}=p_{\text {br }}=438$ Torr c данным вибратором был зафиксирован факт инициации СВЧ пробоя. С этого момента уменьшение $p_{c}$ было прекращено, а вспомогательный разрядник отключен. При этом при данном значении $p_{c}$ и троекратном включении СВЧ импульса пробой воздуха не происходил. Давление $p_{c}$ начинало ступенчато с шагом 3 Torr уменьшаться, и при каждом его значении производилось трехкратное включение СВЧ импульсов. В опытах с данным вибратором лишь при $p_{c}=390$ Torr во втором последовательном СВЧ импульсе разряд был зафиксирован. На рис. 2 крестиком обозначено это значение $p_{c}=p_{\text {br }}$, а рядом в скобках указано, на каком по счету импульсе произошел СВЧ пробой.

Затем длина вибратора уменьшалась на $1 \mathrm{~mm}$, и опытный цикл в указанной последовательности повторялся. И так до минимальной длины вибратора $2 L=50 \mathrm{~mm}$.

Из рис. 2 следует, что зависимость $p_{\mathrm{br}}(2 L)$ при использовании УФ излучения имеет резонансный регулярный характер. При этом данный вибратор инициирует пробой воздуха и при атмосферном давлении в диапазоне $2 L$ от 52 до $56 \mathrm{~mm}$. Зависимость же $p_{\text {br }}(2 L)$ без УФ излучения, хотя и близка к резонансной, существенно ниже „аппроксимированной“ и нерегулярна. В этом случае пробой воздуха при атмосферном давлении вообще не осуществлялся. Кроме того, при конкретных значениях $2 L$ и пороговом давлении $p_{\mathrm{br}}$ инициация пробоя осуществлялась не в каждом из трех СВЧ импульсов.

\section{Обсуждение}

Условие СВЧ пробоя воздуха принято записывать в виде [4]

$$
v_{i} \geq v_{a}+v_{d}+v_{\tau} ; 1 / \mathrm{s},
$$

где $v_{i}$ - частота ионизации молекул воздуха электронными ударами, $v_{a}$ - частота прилипания разрядных электронов к молекулам воздуха, $v_{d}$ - частота их диффузионного ухода из пробойной области и $v_{\tau}-$ частота, характеризующая временну́ю ограниченность СВЧ импульса. В СВЧ поле энергия разрядных электронов $\varepsilon_{e}$ лежит в узком диапазоне значений, близких к $\varepsilon_{e}=1 \mathrm{eV}$. Это позволяет рассчитывать перечисленные величины оценочными выражениями [8]

$$
\begin{gathered}
v_{i}=v_{a}\left(E / E_{\mathrm{cr}}\right)^{5.3} ; 1 / \mathrm{s}, \\
v_{a}=2 \cdot 10^{4} p ; 1 / \mathrm{s},
\end{gathered}
$$

$E$ - амплитуда СВЧ поля;

$$
E_{\mathrm{cr}}=42 p \sqrt{1+\left(\omega / \nu_{c}\right)^{2}} ; \mathrm{V} / \mathrm{cm}
$$

- амплитуда критического поля пробоя, $\omega$ - круговая частота поля,

$$
v_{c}=4 \cdot 10^{9} p ; 1 / \mathrm{s}
$$

— частота столкновений электронов с молекулами воздуха,

$$
v_{\tau}=30 / \tau_{\mathrm{pul}} ; 1 / \mathrm{s}
$$

В этих выражениях, как и в последующих, без специальной оговорки, размерность давления - Torr.

В случае реализации условия (1) поле $E$ в формуле (2) и есть поле пробоя $E_{\mathrm{br}}$. Отсюда требуемое минимальное превышение пробойного поля над критическим в пространственно-ограниченной пробойной области

$$
\xi=E_{\mathrm{br}} / E_{\mathrm{cr}}=\left(1+v_{d} / v_{a}\right)^{1 / 5.3} .
$$

В это выражение на основе оценок, учитывающих рассматриваемые опытные условия, частота $v_{\tau}$ не включена.

Частота диффузионного ухода электронов $v_{d}$ из пробойной области с $E \geq E_{\mathrm{cr}}$ зависит от ее геометрии и условий на границах. Для некоторых простейших случаев в работе [4] даны формулы для расчета значений $v_{d}$.

В работе [10] приведен порядок расчета коэффициента $\xi$ для случая пробоя воздуха, инициированного облучаемым УФ излучением металлическим шариком, помещенным в линейно поляризованный СВЧ пучок. В ней учтено, что поле около полюсов шарика, где вектор $\mathbf{E}_{0}$ перпендикулярен его поверхности, описывается законом

$$
E_{\mathrm{pol}}=E_{0}+2 E_{0} /(1+x / a)^{3},
$$

где коэффициент 2 показывает, во сколько раз амплитуда индуцированного поля на поверхности шарика больше амплитуды исходного поля $E_{0}, x$ - расстояние от полюсной поверхности шарика, а $a-$ его радиус. В настоящей работе показано, что величина $\xi=E_{\mathrm{polmax}} / E_{\mathrm{cr}} \equiv E_{\mathrm{br}} / E_{\mathrm{cr}}$ зависит от параметра

$$
\eta=l_{a} / a=0.84 /(a p),
$$

где с учетом наличия УФ излучения диффузионная длина прилипания электронов $l_{a}=\sqrt{D_{a} / \nu_{a}}=0.84 / p ; \mathrm{cm}$ при коэффициенте амбиполярной диффузии $D_{a}=$ $=1.4 \cdot 10^{4} / p ; \mathrm{cm}^{2} / \mathrm{s}$ и размерности $a-\mathrm{cm}$. Расчет в этой работе коэффициента $\xi$ показал его рост от 1 до 2.5 при увеличении значений параметра $\eta$ от 0 до 0.3 . Таким образом, в реальных ситуациях поле на полюсах шарика, обеспечивающее пробой воздуха $E_{\mathrm{polmax}}=E_{\mathrm{br}}$, может существенно превышать $E_{\mathrm{cr}}$.

В случае цилиндрического вибратора со сферически закругленными концами поле на его полюсах $E_{\text {pol max }}$ может в десятки-сотни раз превышать поле $E_{0}$. В то же время расчеты показывают, что при этом закон „спадания“ индуцированного поля при $x \leq 0.5 a$ практически близок закону (8). Следовательно, при использовании УФ подсветки коэффициент $\xi$ задается, как и для шарика, определенным формулой (9) коэффициентом $\eta$.

Теория расчета пробойного поля $E_{\mathrm{br}}$ в случае вибратора с конически заостренным концом в настоящее 
время не разработана. В то же время очевидно, что, с одной стороны, в соответствии с законами электродинамики индуцированное поле на его конусной поверхности может в еще большее число раз превышать поле $E_{0}$, особенно при приближении к острию конуса. С другой стороны, при приближении к острию закон спадания этого поля будет более резким по сравнению с (8), и влияние диффузионного фактора на процесс пробоя будет более существенным. Следовательно, величина индуцированного поля у конусной поверхности вибратора, обеспечивающая пробой воздуха, может и должна в разы превышать поле $E_{\mathrm{cr}}$.

Воздух условно принято называть электроотрицательным газом. Это связано с тем, что часть его молекул кислорода $\mathrm{O}_{2}$ заряжена отрицательно $\mathrm{O}_{2}^{-}$. В атмосферном воздухе за счет фоновых излучений в результате ионизации его молекул возникает некоторое число свободных электронов [4]

$$
Q_{e}=10(p / 760), 1 /\left(\mathrm{cm}^{3} \mathrm{~s}\right) .
$$

Эти электроны почти „мгновенно“ прилипают к молекулам кислорода в тройных столкновениях $e+\mathrm{O}_{2}+$ $+\mathrm{N}=\mathrm{O}_{2}+\mathrm{N}$, где $\mathrm{N}$ - любая молекула, с частотой прилипания

$$
v_{a 1}=(10 p)^{2}, 1 / \mathrm{s} .
$$

Затем включаются рекомбинационные процессы, и в заключение около 30\% молекул кислорода воздуха оказываются отрицательно заряженными. Их концентрация

$$
n_{\mathrm{O}_{2}^{-}} \approx 2.4 \cdot 10^{18}(p / 760), 1 / \mathrm{cm}^{3}
$$

При включении СВЧ поля на эти молекулы начинает действовать эффективная электрическая сила $F=$ $=q_{e} E / \sqrt{2}$, где $q_{e}=1.6 \cdot 10^{-19}, \mathrm{~K}$ - заряд электрона и на среднем пути до столкновения с другими молекулами $l_{\mathrm{av}}=5 \cdot 10^{3} / p, \mathrm{~cm}$ они набирают дополнительную энергию

$$
\Delta \varepsilon=E q_{e} l_{\mathrm{av}} / \sqrt{2}, \mathrm{~J} .
$$

Если эта энергия близка к энергии отлипания электрона от молекулы кислорода $\varepsilon_{\mathrm{det}}=1 \mathrm{eV}$, то появляется вероятность его отлипания. Таким образом, при большом уровне СВЧ поля отлипание от $\mathrm{O}_{2}^{-}$электронов и без вспомогательного УФ излучения может обеспечить необходимое их начальное наличие в пробойной области.

Формулы (4) и (13) показывают, что в независимости от $p$ для обеспечения этого явления поле $E$ должно быть примерно в 6.7 раз больше $E_{\text {cr. }}$ Однако это значение рассчитано для среднего значения $l_{\mathrm{av}}$. Реально пути до столкновений молекул $\mathrm{O}_{2}^{-}$с другими молекулами воздуха имеют статистический разброс и, следовательно, даже при меньшем поле часть $\mathrm{O}_{2}^{-}$молекул могут обеспечить требуемую величину энергии $\varepsilon_{\text {det }}$.

В качестве ориентира для экспериментальных данных проведем некоторые абсолютные оценки введенных величин.
Как уже указывалось, вибратор со сферически закругленными концами при $2 L=(50-60) \mathrm{mm}$ без УФ подсветки не инициировал СВЧ разряд. Следующие из рис. 2 полученные при УФ подсветке экспериментальные значения $p_{\mathrm{br}}=(200-50)$ Torr. При $2 a=5.6 \mathrm{~mm}$ по (9) они дают значения коэффициента $\eta=0.015-0.06$, что соответствует величинам $\xi=1.15-1.4$. Следовательно, поле в приполюсных областях вибратора с такими длинами лишь ненамного превышает $E_{\text {cr. }}$ В этом случае говорить о возможном отлипании электронов не приходится. Это и фиксирует эксперимент.

Из рис. 2 следует, что экспериментальные значения $p_{\text {br }}$ для вибратора с конически заостренным концом в этом диапазоне значений $2 L$ при наличии УФ подсветки находятся на порядок выше, чем данные для вибратора со сферически закругленными концами. Это и подтверждает тот факт, что индуцированное поле у заостренного конца вибратора весьма значительно. Однако по опытным значениям $p_{\mathrm{br}}$ для конкретных $2 L$ можно рассчитать только величины $E_{\mathrm{cr}}$. По ним нельзя оценить значения коэффициентов $\xi$. Ясно только, что он имеет масштаб нескольких единиц. Это же подтверждает и факт инициации таким вибратором пробоя воздуха без УФ подсветки. Зафиксированный статистический характер этого явления констатирует, что в пробойной области при реализующихся локальных значениях коэффициента $\xi=E_{\text {pol max }} / E_{\text {cr }}$ факт отлипания электронов от молекул $\mathrm{O}_{2}^{-}$является нерегулярным.

Здесь уместно сделать следующее замечание.

При построении опирающихся на процесс „отлипания“ правил расчета коэффициента $\xi$ надо учитывать, что в этом случае „убегание“ электронов из пробойной области будет определяться не амбиполярной, а свободной их диффузией. Коэффициент же свободной диффузии $D_{e}=1.6 \cdot 10^{6} / p ; \mathrm{cm}^{2} / \mathrm{s}$ на два порядка больше коэффициента $D_{a}$. В результате в разрабатываемой теории на порядок возрастет значение коэффициента $\eta$ и определяемое им обеспечивающее пробой воздуха превышение поля $E_{\text {polmax }}$ над полем $E_{\text {cr. }}$ В свою очередь, высокий уровень поля и обеспечивает отлипание электронов от молекул $\mathrm{O}_{2}^{-}$.

\section{Заключение}

Таким образом, экспериментально с использованием ЭМ вибратора с конически заостренным концом удалось осуществить электрический пробой воздуха в квазиоптическом СВЧ пучке с глубоко подкритическим уровнем поля. При этом пробой был осуществлен и без облучения ЭМ вибратора УФ излучением. Сравнительно простая геометрия использованного вибратора позволяет развить соответствующую теорию и сравнить расчетные результаты с опытными данными. В реальных устройствах вибратор может иметь и другую геометрию. Например, он может быть более заостренным [7] или выполнен из провода существенно меньшего диаметра и осуществлять пробой воздуха при больших давлениях и меньших уровнях исходного поля. 
Выполненные эксперименты дают основание предположить, что в электрических разрядных процессах существенную роль может играть процесс отлипания электронов от молекул кислорода воздуха в интенсивном электрическом поле - и не только СВЧ диапазона. Это может помочь анализировать, например, различные необъяснимые пока процессы в грозовых атмосферных явлениях.

\section{Список литературы}

[1] Esakov I.I., Grachev L.P., Khodataev K.V., Vinogradov V.A., Van Wie David M. // $44^{\text {rd }}$ AIAA Aerospace Sciences Meeting and Exhibit (Reno, Nevada, USA, 2006). AIAA-2006-1212.

[2] Esakov I., Grachev L., Khodataev K., Vinigradov V., Van Wie D. // $46^{\text {rd }}$ AIAA Aerospace Sciences Meeting and Exhibit. Reno, Nevada, USA, 2008. AIAA-2008-1403.

[3] Bychkov D.V., Esakov I.I., Grachev L.P., Khodataev K.V., Van Wie D.M. $/ / 47^{\text {rd }}$ AIAA Aerospace Sciences Meeting and Exhibit. Orlando, USA, 2009. AIAA-2009-1551.

[4] Райзер Ю.П. Физика газового разряда. М.: Наука, 1987. $591 \mathrm{c}$.

[5] Грачев Л.П., Есаков И.И., Мишин Г.И., Ходатаев К.В. // ЖТФ. 1995. Т. 65. Вып. 7. С. 60-67.

[6] Александров К.В., Грачев Л.П., Есаков И.И., Раваев А.А., Северинов Л.Г., Яковлев А.Ю. // ЖТФ. 2016. Т. 86. Вып. 4. C. 28-33.

[7] Esakov I., Grachev L., Khodataev K., Van Wie D. // 49 ${ }^{\text {th }}$ AIAA Aerospace Sciences Meeting. Orlando, Florida, USA, 2012. AIAA-2011-1151.

[8] Грачев Л.П., Есаков И.И., Александров К.В., Раваев А.А., Северинов Л.Г., Ходатаев К.В. Газовый электрический разряд в квазиоптическом СВЧ пучке. М.: АО МРТИ РАН, 2015. $187 \mathrm{c}$.

[9] Александров К.В., Грачев Л.П., Есаков И.И. // ЖТФ. 2007. Т. 77. Вып. 12. С. 26-30.

[10] Грачев Л.П., Есаков И.И., Ходатаев К.В., Цыпленков В.В. // Физика плазмы. 1992. Т. 18, Вып. 3. С. 411-413. 